\title{
Tumbleweeds and airborne gravitational noise sources for LIGO
}

\author{
Teviet Creighton \\ Department of Physics, University of Wisconsin-Milwaukee, P.O. Box 413, Milwaukee, Wisconsin 53201
}

(Dated: October 29, 2018)

\begin{abstract}
The test masses in gravitational-wave detectors will be sensitive not only to astrophysical gravitational waves, but also to the fluctuating Newtonian gravitational forces of moving masses in the ground and air around the detector. These effects are often referred to as gravity gradient noise. This paper considers the effects of gravity gradients from density perturbations in the atmosphere, and from massive airborne objects near the detector. These have been discussed previously by Saulson, who considered the effects of background acoustic pressure waves and of massive objects moving smoothly past the interferometer; the gravity gradients he predicted would be too small to be of serious concern even for advanced interferometric gravitational-wave detectors. In this paper I revisit these phenomena, considering transient atmospheric shocks, and estimating the effects of sound waves or objects colliding with the ground or buildings around the test masses. I also consider another source of atmospheric density fluctuations: temperature perturbations that are advected past the detector by the wind. I find that background acoustic noise and temperature fluctuations still produce gravity gradient noise that is below the noise floor even of advanced interferometric detectors, although temperature perturbations carried along non-laminar streamlines could produce noise that is within an order of magnitude of the projected noise floor at $10 \mathrm{~Hz}$. A definitive study of this effect may require better models of the wind flow past a given instrument. I also find that transient shockwaves in the atmosphere could potentially produce large spurious signals, with signal-to-noise ratios in the hundreds in an advanced interferometric detector. These signals could be vetoed by means of acoustic sensors outside of the buildings. Massive wind-borne objects such as tumbleweeds could also produce gravity gradient signals with signal-to-noise ratios in the hundreds if they collide with the interferometer buildings, so it may be necessary to build fences preventing such objects from approaching within about $30 \mathrm{~m}$ of the test masses.
\end{abstract}

PACS numbers: $04.80 . \mathrm{Nn}, 95.55 . \mathrm{Ym}$

\section{INTRODUCTION}

Interferometric detectors such as LIGO and VIRGO rely on exquisite sensitivity to the positions of hanging test masses in order to detect the perturbations of passing gravitational radiation. The sensitivity is so great that the measurements can also be affected by fluctuations in the local Newtonian gravitational field, which create tiny accelerations of the mass. This noise source, known as gravity gradient noise or Newtonian gravitational noise, is caused by the near-field gravity of masses moving near the interferometer, and is not to be confused with the far-field propagating gravitational waves that the instruments are intended to measure.

Gravity gradient noise has the potential to be quite insidious, since it cannot be shielded by improvements to the test-mass vibrational isolation. The only effective way to eliminate gravity gradient noise is to eliminate the moving masses that create the perturbing fields. Fortunately, though, the strongest perturbations to the local gravitational field are at frequencies well below the detectors' pass-bands. Since the proposed terrestrial interferometric detectors all have sensitivity cutoffs around $3 \mathrm{~Hz}$ or higher, we need only worry about noise sources that can perturb the local gravity field on timescales less than about 0.3 seconds. Most of the noise sources that I consider are motivated by the expected sensitivity of advanced LIGO interferometers, which were originally projected to have a low-frequency cutoff around $10 \mathrm{~Hz}$, and instrumental noise of $S_{h} \sim 2 \times 10^{-45} \mathrm{~Hz}^{-1}$ in the gravitational-wave signal output at that frequency [1] [13. Although changes in instrumentation technology will modify the ultimate sensitivity goals of LIGO, this "standard" advanced LIGO noise level is a good reference point when considering new noise sources. Also, as pointed out below, gravity gradient noise will make it difficult to push the detector noise much below this level at $10 \mathrm{~Hz}$, regardless of improvements to the interferometers.

Saulson [2] was the first to estimate the effect of gravity gradient noise on terrestrial interferometric detectors, considering the effects of seismic waves passing through the earth and of sound waves in the air. In both cases he found the spectral density of noise in the interferometer path-length difference to be less than $10^{-39} \mathrm{~m}^{2} \mathrm{~Hz}^{-1}$ around $10 \mathrm{~Hz}$, corresponding to noise in the gravitational-wave signal at levels less than $10^{-46} \mathrm{~Hz}^{-1}$ for a $4 \mathrm{~km}$ interferometer. By comparison, this is significantly less than the noise floor of $\sim 2 \times 10^{-45} \mathrm{~Hz}^{-1}$ that advanced LIGO interferometers expect to achieve at $10 \mathrm{~Hz}$. More recently, a detailed analysis [3] has been made of seismic gravity gradient noise; this study indicated that seismic gravity gradient noise would be within a factor of 2 of the advanced LIGO noise floor at $10 \mathrm{~Hz}$ for most times, and could actually exceed this noise floor during seismically noisy times, making seismic gravity gradients a significant barrier to improvements in low-frequency sensitivity. It therefore 
seems prudent to revisit the issue of atmospheric gravity gradients as well.

In Sec. II I consider gravity gradients caused by atmospheric pressure perturbations - the same noise source considered by Saulson. Attention is paid, however, to the effects of the ground, and of buildings that reduce the pressure noise in the immediate vicinity of the interferometer test masses. Nonetheless, I find that these tend only to weaken the gravity gradient noise in the pass-bands of interferometric detectors, reinforcing the conclusion that this noise source is not of any great concern.

A much stronger source of high-frequency density perturbations in the atmosphere is the presence of temperature fluctuations, which are advected past a detector by the wind. In Sec. III I analyze this as a potential source of gravity gradient noise. However, I find that while small-scale temperature perturbations can produce highfrequency temperature fluctuations at any given point, they do not produce the same high-frequency fluctuations in the test mass position, since a given pocket of warm or cool air will affect the test mass gravitationally over the entire time that it is in the vicinity of the test mass, which is on the order of seconds. This produces a cutoff in the noise spectrum above a few tenths of a $\mathrm{Hz}$. The presence of turbulent vortices around the interferometer buildings can increase the high-frequency component, but still probably not enough to show up in the gravitational-wave noise spectrum.

In Secs. IV and V I turn away from sources of background noise to consider possible sources of transient gravity gradient signals that might be detected as spurious events in the gravitational-wave instruments. Sec. IV extends the analysis in Sec. II to look at the effects of atmospheric shockwaves, such as might be generated by an explosion or supersonic aircraft. I find that sources such as these can indeed produce detectable signals that might be interpreted spuriously as gravitational-wave events. However, such signals would easily be vetoed using acoustic monitors outside the interferometer buildings.

Sec. $\mathrm{V}$ analyzes the gravity gradients produced by individual objects, such as wind-borne debris, moving around outside the interferometer buildings. Saulson considered this effect for the case of objects moving with fairly uniform velocity, but typically, in order to produce significant signal above $3 \mathrm{~Hz}$, an object's motion must be changing on timescales of less than 0.3 seconds. In particular, I find that objects colliding with the interferometer buildings produce much stronger signals than objects simply passing by the buildings. As an example, tumbleweeds at the Hanford LIGO facility will be a steady source of spurious signals in advanced detectors if they are allowed to collide with the end stations. Preventing such signals requires shielding a region of a few tens of meters around the end station, screening any wind-borne debris that masses more than a few hundred grammes.

Sec. VI presents some concluding remarks, including recommendations to the gravitational-wave experimental groups and possible directions for further research.

\section{ATMOSPHERIC PRESSURE WAVES}

Pressure perturbations are the only type of atmospheric gravity gradient noise considered by Saulson [2. The derivation in this section gives largely the same result as his.

Consider a plane pressure wave with frequency $f$ propagating through a homogeneous airspace at some sound speed $c$. If the fractional pressure change $\delta p / p$ is small, it will induce an adiabatic density change $\delta \rho / \rho=\delta p / \gamma p$, where $\gamma \approx 1.4$ is the ratio of heat capacities at constant pressure and constant temperature for air at normal temperatures. The gravitational acceleration produced by this wave in the direction of propagation $\mathbf{e}_{z}$ is:

$$
g_{z}(t)=\int \frac{G z \delta \rho}{r^{3}} d V=\frac{2 G \rho c}{\gamma p f} \delta p(t+1 / 4 f),
$$

where $\rho \approx 1.3 \mathrm{~kg} \mathrm{~m}^{-3}$ and $p \approx 10^{5} \mathrm{~N} \mathrm{~m}^{-2}$ are the ambient air density and pressure, and $\delta p(t)$ is the pressure perturbation measured at the same point as the acceleration is being measured. By symmetry, there is no acceleration transverse to the wave.

Now consider sound waves in the vicinity of the interferometer. First, since the interferometer is only sensitive to motions of the test mass parallel to the arms, the gravitational acceleration is reduced by a factor $\cos \theta$, where $\theta$ is the angle between the propagation direction and the interferometer arm.

Second, the interferometer test mass is inside a building, which can in principle be used to suppress noise within a distance $r_{\min }$ of the test mass. Roughly speaking, this results in a high-frequency cutoff factor $C\left(2 \pi f r_{\min } / c\right)$, where the function $C(x)$ depends on the precise shape of the building, the manner in which it reflects sound waves, and many other factors, but is normally close to 1 for $x \lesssim 1$. For instance, if one simply removes from the volume integral in Eq. (1) a cylinder with length and diameter both $2 r_{\text {min }}$ aligned with the $z$-axis, then $C(x) \sim 1$ for $x \lesssim 1$, but oscillates with an amplitude of $\sim 0.3$ for $x \gtrsim 1$. This function is shown in Fig. 1. The constant-amplitude oscillations of $C(x)$ for large $x$ reflect the assumption that the sound wave has a coherence length much longer than the building size, so the field between the two ends of the excluded cylinder is generated almost entirely by the first halfwavelength beyond each cap. Realistically, the actual high-frequency behaviour of $C(x)$ will depend on how the sound waves bend and scatter around the building; however, this should not change the order of magnitude of $C(x)$. The behaviour shown in Fig. 1 is therefore probably a good estimate of the true cutoff function for $x \lesssim 1$, and a reasonable order of magnitude estimate for $x \gtrsim 1$. For the LIGO end stations, $r_{\text {min }}$ is of order 5 metres, giving $x \sim f /(10 \mathrm{~Hz})$; the factor will not be too far off 


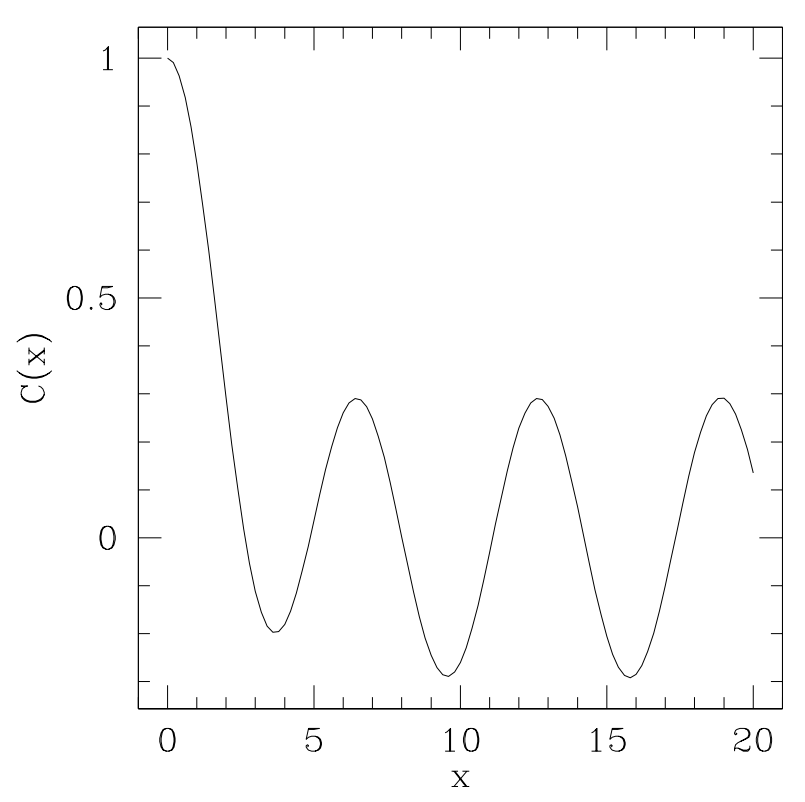

FIG. 1: Plot of the factor $C(x)$ indicating the reduction in sonic gravity gradient noise due to setting the density perturbations to zero within a cylinder of diameter and length $2 r_{\text {min }}$ centered on the test mass (and aligned with the wave), versus $x=2 \pi r_{\min } / \lambda$ where $\lambda$ is the wavelength. The oscillatory behaviour above $x=1$ results from the ends of the cylinder coming in and out of phase with each other. A more accurate model of the scattering of sound waves off an interferometer end station building would modify the behaviour of $C(x)$ for $x>1$, but probably not change its magnitude significantly.

for the frequencies of greatest interest. More precise estimates would depend on the specific architectural details of a particular facility.

Third, the interferometer is on the ground, not in homogeneous empty space. For simplicity I assume that the waves are almost entirely reflected off the ground; I will justify this assumption in Sec. II A. In this case the gravity gradient in directions parallel to the ground contributed by the reflected wavefront is the same as if the wavefront were extended below ground, while the pressure perturbations measured by detectors near the ground (much less than a wavelength) will be doubled. The acceleration experienced by an interferometer test mass is therefore:

$$
g_{z}(t)=\frac{G \rho c}{\gamma p f} \cos (\theta) C\left(2 \pi f r_{\min } / c\right) \delta p(t+1 / 4 f) .
$$

The gravitational wave signal $h(t)$ in the interferometer is related to the acceleration of one of the test masses by $\ddot{h}(t)=g(t) / L$, or in frequency space $\tilde{h}(f)=$ $(2 \pi f)^{-2} \tilde{g}(f) / L$, where $L$ is the length of the interferometer arm. Thus:

$$
\tilde{h}(f)=\frac{G \rho c}{4 \pi^{2} \gamma p L f^{3}} \cos (\theta) C\left(2 \pi f r_{\min } / c\right) i \widetilde{\delta p}(f) .
$$

Assuming stationary noise, the one-sided spectral density
$S_{h}(|f|)$ is given by $\left\langle\tilde{h}(f) \tilde{h}\left(f^{\prime}\right)^{*}\right\rangle=S_{h}(|f|) \delta\left(f-f^{\prime}\right)$, where $\langle\ldots\rangle$ denotes an expectation over all random phases of all plane wave modes contributing to the noise, and * denotes complex conjugation. Taking mode amplitudes and directions to be uncorrelated, this gives:

$$
S_{h}(|f|)=\left[\frac{G \rho c}{4 \pi^{2} \gamma L f^{3}} C\left(2 \pi f r_{\min } / c\right)\right]^{2}\left\langle\cos ^{2} \theta\right\rangle \frac{S_{p}(|f|)}{p^{2}},
$$

where $S_{p}(|f|)$ is the acoustic noise spectral density measured outside the building in the vicinity of a particular test mass. Since the two test masses in an arm are many wavelengths apart, their noise will be uncorrelated, and will thus add in noise power. Similarly, the noise from the two arms will add in power. (Actually this is a bit of an overestimate, since the noise in the motion of the test masses at the corner station will be somewhat correlated.) Noting that $\left\langle\cos ^{2} \theta\right\rangle=1 / 3$, one finds that the total noise in the gravitational wave signal is:

$S_{h}(|f|)=\left(\frac{G \rho c}{4 \pi^{2} \gamma L}\right)^{2} \frac{1}{3 f^{6} p^{2}} \sum_{i=1}^{4} C\left(2 \pi f r_{\min }^{(i)} / c\right)^{2} S_{p}^{(i)}(|f|)$,

where $i$ denotes a particular test mass in the interferometer, $r_{\text {min }}^{(i)}$ is the dead air radius about the $i$ th test mass, and $S_{p}^{(i)}(|f|)$ is the acoustic noise spectrum measured outside the building enclosing that test mass.

Infrasound noise spectra should be taken at the actual interferometer sites, but one can make estimates based on typical terrestrial atmospheric noise. An empirical study [⿶] collected 256 power spectra of $1-16 \mathrm{~Hz}$ infrasound data from a rural forest $50 \mathrm{~km}$ from New York City over a period of months, and found that the average noise spectrum $S_{p}(f)$ was relatively flat at $6-16 \mathrm{~Hz}$, though with widely varying amplitude: $25 \%$ of the spectra had noise under $\sim 100 \mathrm{nbar}^{2} / \mathrm{Hz}, 50 \%$ under $\sim 300 \mathrm{nbar}^{2} / \mathrm{Hz}$, and $75 \%$ under $\sim 1000 \mathrm{nbar}^{2} / \mathrm{Hz}$. I use Eq. (5) to compute the corresponding noise in the LIGO detector. Assuming that the end masses (with $r_{\min } \sim 5 \mathrm{~m}$ ) dominate the contribution to the gravity gradient noise and contribute equally, the noise in the gravitational-wave signal around $10 \mathrm{~Hz}$ is:

$$
\begin{aligned}
S_{h}(|f|) \sim & \left(6 \times 10^{-48} \mathrm{~Hz}^{-1}\right)\left(\frac{f}{10 \mathrm{~Hz}}\right)^{-6}\left(\frac{S_{p}(|f|)}{1000 \mathrm{nbar}^{2} / \mathrm{Hz}}\right) \\
& \times C(f / 10 \mathrm{~Hz}) .
\end{aligned}
$$

The results are plotted in Fig. 2, using infrasound power spectra read off of Fig. 3 of 迎.

Even the third-quartile power spectrum is between two and three orders of magnitude below the expected noise floor of $2 \times 10^{-45} \mathrm{~Hz}^{-1}$ at $10 \mathrm{~Hz}$ projected for advanced LIGO interferometers. Thus ambient infrasound is probably a negligible effect for determining the noise floor for most interferometric gravitational-wave detectors. Nonetheless the issue cannot be completely resolved without infrasonic noise data from the actual interferometer sites. 


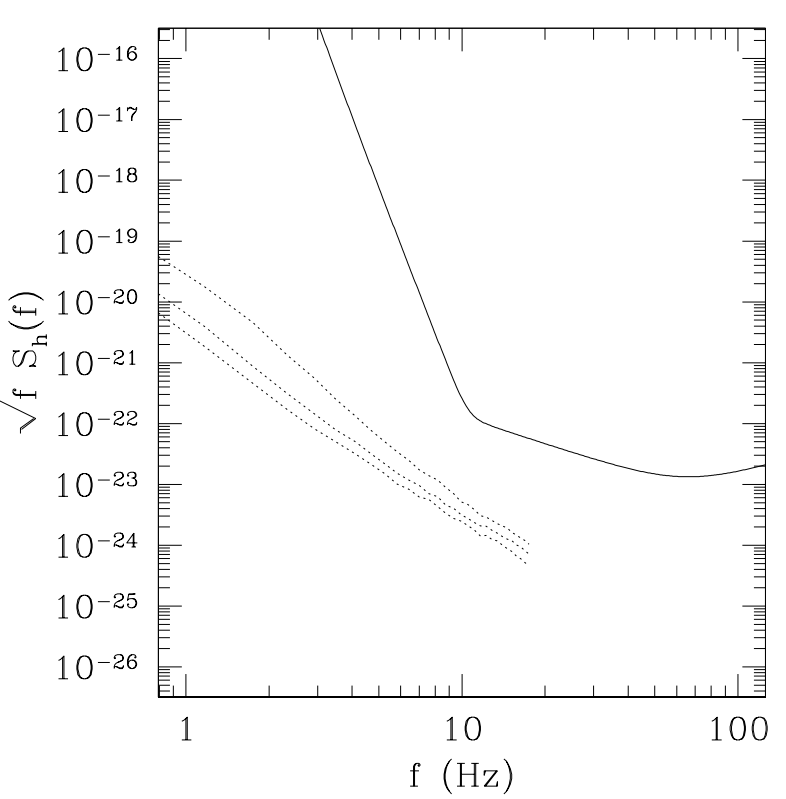

FIG. 2: Plot of dimensionless strain noise $\sqrt{f S_{h}(f)}$ versus frequency $f$ for infrasonic atmospheric gravity gradients. The solid curve is the projected noise floor for advanced LIGO detectors; the dotted curves are the first, second, and third quartiles of noise produced by gravity gradients from ambient pressure waves. Data for the infrasonic noise power are taken from Fig. 3 of 4 . Clearly the pressure waves would not contribute significantly to LIGO noise in any frequency range.

\section{A. Ground absorption}

In the derivation above I treated the sound waves as being reflected off the ground. Now consider what happens if a sound wave is absorbed by the ground. The energy flux in a traveling compression wave is $C^{3 / 2} \rho^{-1 / 2}\left\langle(\delta x / x)^{2}\right\rangle$, where $C$ is the compression modulus of the medium $\left(C_{\text {air }}=\gamma p_{\text {air }}\right)$, and $\left\langle(\delta x / x)^{2}\right\rangle$ is the average squared dimensionless compression factor over a wave cycle. The gravity gradient noise induced by such a wave goes as $c^{2}\left\langle(\delta \rho)^{2}\right\rangle$, where $\left\langle(\delta \rho)^{2}\right\rangle \sim \rho\left\langle(\delta x / x)^{2}\right\rangle$, and $c=\sqrt{C / \rho}$ is the wave speed in that medium. Therefore, if a sound wave is completely absorbed by the ground, the resulting ground motions will produce gravity gradient noise contributions in the ratio:

$$
\frac{S_{h}^{\text {(ground) }}}{S_{h}^{\text {(air) }}}=\left(\frac{\rho_{\text {ground }}}{\rho_{\text {air }}}\right)^{3 / 2}\left(\frac{C_{\text {ground }}}{C_{\text {air }}}\right)^{-1 / 2} .
$$

Now for $C_{\text {air }}=\gamma p_{\text {air }}=1.4 \times 10^{5} \mathrm{~N} \mathrm{~m}^{-2}, C_{\text {ground }} \sim 3 \times$ $10^{8} \mathrm{~N} \mathrm{~m}^{-2}, \rho_{\text {air }}=1.3 \mathrm{~kg} \mathrm{~m}^{-3}, \rho_{\text {ground }} \sim 1.8 \times 10^{3} \mathrm{~kg} \mathrm{~m}^{-3}$, the ratio turns out to be of order $10^{3}$; that is, if sound waves were completely absorbed by the ground, the resulting ground vibrations would produce gravity gradient noise levels about 1000 times greater than the atmospheric gravity gradients. However, it was shown in [3] that seismic gravity gradients are only just large enough to worry about. So the only way that atmospheric gravity gradients can be larger or of the same order as seismic gravity gradients is if the waves are mostly reflected off of the ground. This was one of the assumptions used in deriving Eq. (2).

\section{ATMOSPHERIC TEMPERATURE PERTURBATIONS}

The largest small-scale atmospheric density perturbations are caused not by pressure waves but by temperature perturbations. As heat is transported up through a convective atmospheric layer, convective turbulence mixes pockets of warm and cool air to form temperature perturbations on all lengthscales down to a few millimetres. On the timescales of interest (less than a second) these perturbations are effectively "frozen" into the airmass, while pressure differences disperse rapidly in the form of sound waves. Perturbations in the air density $\rho \propto p / T$ are therefore caused predominantly by the temperature perturbations, which are typically several orders of magnitude larger than the pressure perturbations. Although they are frozen into the airmass, these temperature perturbations can cause rapid time-varying density fluctuations $\delta \rho=-\rho \delta T / T$ as the wind carries them past a point in space. This is the primary source of "seeing" noise that affects optical astronomy.

The appendix to this chapter gives a rigorous mathematical derivation of the gravity gradient noise spectrum due to these temperature perturbations. This section gives a qualitative derivation that reproduces the final result to order of magnitude.

The gravity gradient signal at some frequency $f$ is caused by pockets of warm or cool air with some lengthscale $l$ being advected past the interferometer test mass at a speed $v$, where $l \sim v / 2 \pi f$. Consider a single such pocket of air with a temperature perturbation $\delta T$ away from the ambient temperature $T$. The gravitational acceleration produced in the instrument as a function of time $t$ is $g_{x}(t)=G \rho l^{3}(\delta T / T) x(t) r^{-3}(t)$, where $\rho$ is the ambient air density, $r(t)$ is the distance of the air pocket from the test mass as it is blown past, and $x(t)$ is this distance projected onto the axis of the interferometer arm. This geometry is sketched in Fig. 3 .

Now in general, the noise power spectral density in any quantity $a$ due to a background of independent, uncorrelated events is $S_{a}(|f|)=(2 / \Delta t)|\tilde{a}(f)|^{2}$, where $\tilde{a}(f)$ is the Fourier spectrum from a single event and $\Delta t$ is the spacing between events. Assuming uncorrelated pockets of air, then independent pockets of air arrive along any given streamline at intervals $\Delta t \sim l / v$, and streamlines separated by more than $l$ add noise incoherently (i.e., add linearly in power). This gives the following noise spectrum:

$$
S_{g}(|f|) \sim \frac{2 l}{v} \int_{\{S\}} \frac{d A}{l^{2}}\left(\frac{G \rho}{T}\right)^{2} \delta T^{2}(l)\left|\tilde{G}_{S}(f)\right|^{2},
$$




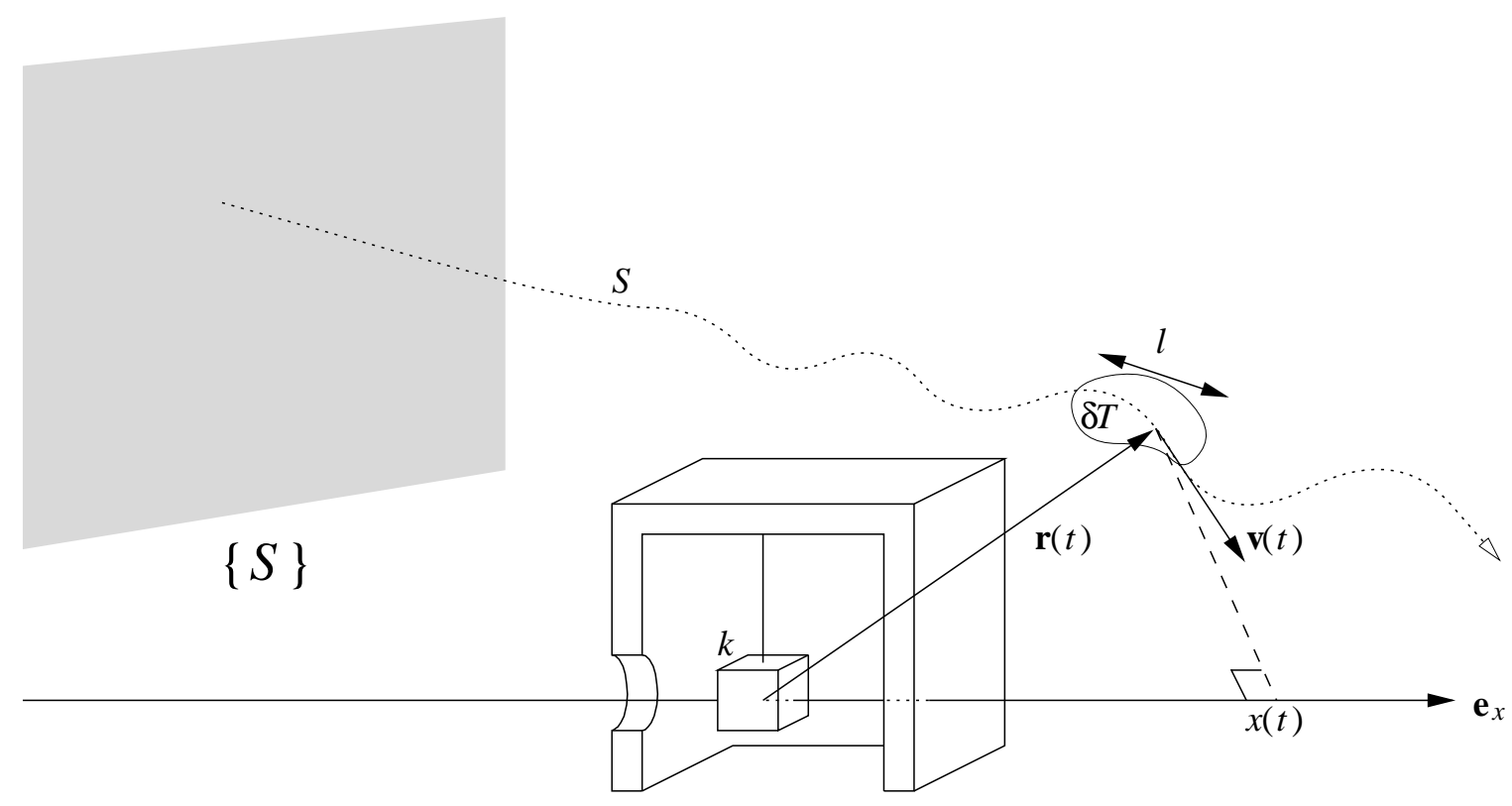

FIG. 3: Schematic of a pocket of air with temperature perturbation $\Delta T$ over a lengthscale $l$, being advected past a test mass $k$ along a streamline $S$. The pocket has an instantaneous velocity $\mathbf{v}(t)$ and position $\mathbf{r}(t)$ relative to the test mass, and $x(t)$ is the projection of $\mathbf{r}(t)$ onto the axis of the interferometer arm. $\{S\}$ denotes a reference plane intersecting orthogonally with all streamlines $S$.

where $\int_{\{S\}}$ denotes an integral over a plane crossing all streamlines $S$, and $\tilde{G}_{S}(f)$ is the Fourier transform of the function $G(t)=x(t) / r^{3}(t)$ taken along a given streamline. The quantity $\delta T^{2}(l)$ is the average squared temperature difference between points a distance $l$ apart. Turbulent mixing theory, as well as actual micrometeorological measurements, predict a power-law behaviour for small separations: $\delta T^{2}(l) \sim c_{T}^{2} l^{p}$, where $p$ is typically $2 / 3$. This applies for horizontal separations $l$ up to of order 50 times the height of a given air pocket above the ground [5]. For streamlines more than a metre or so above ground level, then, this behaviour for $\delta T^{2}$ should be good out to distances $l \sim 50 \mathrm{~m}$, corresponding to frequencies $\gtrsim 0.2 \mathrm{~Hz}\left(v / 10 \mathrm{~m} \mathrm{~s}^{-1}\right)$.

Eq. (8) gives the gravity gradient noise on a given test mass in the interferometer. Denoting the test masses by the index $k=1 \ldots 4$, and assuming that each test mass contributes independently to the noise in the gravitational-wave signal $h$, one has $S_{h}(|f|)=$ $(2 \pi f)^{-4} L^{-2} \sum_{k} S_{g}(|f|)$. Combining this with Eq. (8) and the relation $l \sim v / 2 \pi f$ yields:

$$
\begin{aligned}
S_{h}(|f|) \sim & 2\left(\frac{G \rho}{L T}\right)^{2} c_{T}^{2}(2 \pi f)^{-(p+7)} v^{p+4} \\
& \times \sum_{k} \int_{\{S\}} d A\left|\tilde{G}_{S, k}(f)\right|^{2} .
\end{aligned}
$$

The more rigorous analysis in the appendix (Sec. A) gives an expression with roughly the same form, but covers the factors of order unity, and also accounts for the fact that wind speed can vary along a streamline and between streamlines. The more accurate formula is:

$$
\begin{aligned}
S_{h}(|f|)= & 2 \pi^{2}\left(\frac{G \rho}{L T}\right)^{2} c_{T}^{2}(2 \pi f)^{-(p+7)} \sin (p \pi / 2) \Gamma(p+2) \\
& \times \sum_{k} \int_{\{S\}} \tilde{F}_{S, k}(f)^{*} \tilde{G}_{S, k}(f) w d A
\end{aligned}
$$

where $w$ is the wind speed of the streamline as it crosses the plane of integration, and $\tilde{F}_{S, k}(f)$ and $\tilde{G}_{S, k}(f)$ are Fourier transforms of functions $F_{S, k}(t)$ and $G_{S, k}(t)$ describing the motion of a point along a streamline $S$ past a test mass $k$, of the form:

$$
\begin{aligned}
& F(t)=\frac{x(t)}{r(t)^{3}} v(t)^{p+3}, \\
& G(t)=\frac{x(t)}{r(t)^{3}} .
\end{aligned}
$$

It is worth noting that the frequency structure of $S_{h}(|f|)$ depends on the time behaviour of the functions $x(t)$ and $r(t)$ describing the position of a point on a streamline relative to a test mass, which can be some distance away. The minimum distance $r_{\min }$ from the test mass to the passing air is thus an additional important scale in the problem: if $x(t)$ and $r(t)$ change significantly only on timescales $\sim r_{\min } / v$, then the noise spectrum will be cut off at frequencies $\gtrsim v / 2 \pi r_{\text {min }}$.

By comparison, the temperature noise spectrum $S_{T}(|f|)$ measured at a point depends only on the local properties of the atmosphere at that point. Applying similar order-of-magnitude arguments, one can write $S_{T}(|f|) \sim(2 l / v) c_{T}^{2} l^{p}|\tilde{H}(f)|^{2}$, where $H(t) \sim 1$ 
for $|t| \lesssim l / v$ and 0 otherwise. At high frequencies $\gtrsim 0.2 \mathrm{~Hz}\left(v / 10 \mathrm{~m} \mathrm{~s}^{-1}\right)$ the system involves only one lengthscale $l$, giving the spectrum a power-law dependence:

$$
S_{T}(|f|) \sim 2 c_{T}^{2} v^{p}(2 \pi f)^{-(p+1)} .
$$

This is the same, to order of magnitude, as the exact result given in Eq. (A3).

\section{A. Uniform airflow}

The gravity gradient noise is easy to compute from Eq. (10) for the case of uniform airflow parallel to the ground with some constant velocity $\mathbf{v}$. Placing the reference plane orthogonal to $\mathbf{v}$ and passing through the test mass, the equations of motion for a given streamline past the test mass become quite simple: $v(t)=w=v$, $x(t)=v t \cos \theta+r_{0} \sin \theta \cos \psi, r(t)=\left(r_{0}^{2}+v^{2} t^{2}\right)^{1 / 2}$, where $r_{0}$ is the distance from the test mass to the nearest point on the streamline, and $r_{0} \cos \psi$ is the projection of this distance onto the ground. The geometry of the airflow is shown in Fig. 1. It is easy to show that:

$$
\begin{aligned}
& \tilde{G}(f)=-\frac{4 \pi f}{v^{2}} {\left[i \cos \theta K_{0}\left(2 \pi f r_{0} / v\right)\right.} \\
&\left.\quad+\sin \theta \cos \psi K_{1}\left(2 \pi f r_{0} / v\right)\right], \\
& \tilde{F}(f)=v^{p+3} \tilde{G}(f),
\end{aligned}
$$

where $K_{0}$ and $K_{1}$ are moddified Bessel functions of the second kind of orders 0 and 1 . I perform the integral over the above-ground half of the reference plane, out from some radius $r_{\min }$ that is roughly the closest distance that the outside air can approach the test mass. This gives a noise contribution from a single test mass equal to:

$$
\begin{aligned}
S_{h}(|f|)= & 8 \pi^{2} \cos (\pi[p-1] / 2) \Gamma(p+2)\left(\frac{G \rho r_{\min }}{T L}\right)^{2} \\
\times & c_{T}^{2}\left(\frac{v}{2 \pi f}\right)^{p}(2 \pi f)^{-5}\left\{\cos ^{2} \theta\left[K_{0}^{2}(x)-K_{1}^{2}(x)\right]\right. \\
& \left.+\frac{1}{2} \sin ^{2} \theta\left[K_{1}^{2}(x)-K_{0}(x) K_{2}(x)\right]\right\}
\end{aligned}
$$

where $x=2 \pi f r_{\min } / v$.

For typical values $v \sim 10 \mathrm{~m} / \mathrm{s}$ and $r_{\min } \sim 5 \mathrm{~m}$, at frequencies above $10 \mathrm{~Hz}$ or so, one has $x \sim 30$ or more, well into the exponentially damped regime of the Bessel functions. Even for gale-force winds of $30 \mathrm{~m} / \mathrm{s}$ or so, the argument $x$ of the Bessel functions will still be of order 10 or more. The asymptotic expansions of $K_{0}, K_{1}$, and $K_{2}$ give $K_{0}^{2}(x)-K_{1}^{2}(x) \sim K_{1}^{2}(x)-K_{0}(x) K_{1}(x) \sim \pi e^{-2 x} /\left(2 x^{2}\right)$. Also, I note that the total noise will be dominated by the contribution from the two end stations, which have smaller $r_{\text {min }}$ than the corner station. So the total noise in the interferometer for uniform airflow is:

$$
\begin{aligned}
S_{h}(|f|)= & (2 \pi)^{3} \cos (\pi[p-1] / 2) \Gamma(p+2)\left(\frac{G \rho}{T L}\right)^{2} c_{T}^{2} \\
& \times\left(\frac{v}{2 \pi f}\right)^{p}(2 \pi f)^{-5} e^{-4 \pi f r_{\min } / v}
\end{aligned}
$$

I consider "typical" values of $p=2 / 3, \rho=1.3 \mathrm{~kg} \mathrm{~m}^{-3}$, $T=300 \mathrm{~K}, L=4000 \mathrm{~m}$, and $r_{\min }=5 \mathrm{~m}$. The parameters $v$ and $c_{T}^{2}$ can vary on a minute-by-minute basis, and should really be measured at the site of a given interferometer. However, $c_{T}^{2} \sim 0.2 \mathrm{~K}^{2} \mathrm{~m}^{-2 / 3}$ is a typical daytime peak temperature fluctuation index [6], and $v \sim 20 \mathrm{~m} / \mathrm{s}$ might be typical of a fairly windy day. At frequencies around $10 \mathrm{~Hz}$, this gives a noise spectrum of:

$S_{h}(|f|) \sim\left(1.6 \times 10^{-40} \mathrm{~Hz}^{-1}\right)\left(\frac{f}{10 \mathrm{~Hz}}\right)^{-23 / 3} 10^{-14(f / 10 \mathrm{~Hz})}$.

The two dotted lines in Fig. 7 show the gravity gradient noise spectra computed from Eq. (18) for wind speeds of $10 \mathrm{~m} / \mathrm{s}$ and $30 \mathrm{~m} / \mathrm{s}$.

Clearly the exponential cutoff makes this a negligible source of noise for LIGO or similar detectors. Physically this cutoff arises from the fact that the gravity from a particular temperature perturbation passing near the end station will affect the test mass coherently over the second or so that it takes to travel the width of the end station. Thus, even though the temperature noise spectrum has a high-frequency power law tail (reflecting the fact that temperature perturbations exist on all lengthscales), the gravity gradient signal will have this much sharper exponentially cut-off tail.

\section{B. Potential flow near the end station}

As described above, uniform airflow is not likely to produce much atmospheric gravity gradient noise in the pass-band of interferometric detectors, since the shortest timescale over which the gradients change is of order the wind crossing time of the interferometer buildings. However, if an air pocket could be made to accelerate over shorter timescales, it might produce a stronger gravity gradient signal at high frequencies.

One possibility is the acceleration of the air as it is forced up and around the wall of an end station: streamlines that approach the ground-wall corner of the end station can have curvature scales much shorter than the building size. Treating the flow as incompressible and vorticity-free, the resulting velocity field near the corner is (p. 27 of $[7]$ ):

$$
v_{x}=-2 A X, \quad v_{z}=2 A Z,
$$

where $A=v / 2 R$ is a constant, and $R, X$, and $Z$ are measured from the corner. This approximation is clearly only good near the corner, since it gives velocity increasing monotonically with radius; one would expect $v$ to approach the free-streaming airspeed $V$ at a distances $R_{m}$

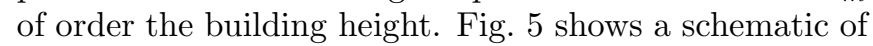
the airflow near the corner.

It is clear from Eq. (19) that, although the streamlines are sharply curved near the corner of the end station, the advection speed is smaller in direct proportion. The shortest timescale over which the motion can change is 


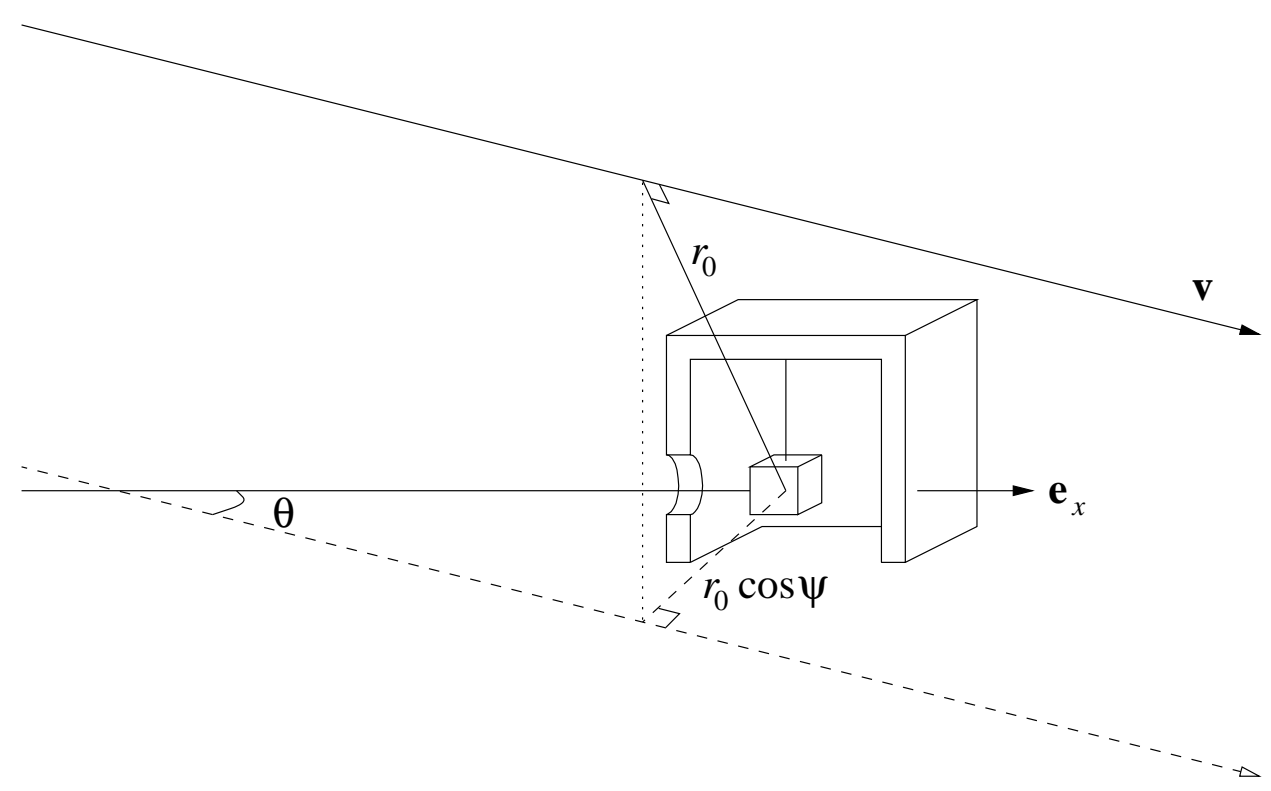

FIG. 4: Schematic of a uniform airflow streamline passing an interferometer corner station. $\theta$ is the angle between the airflow and the axis of the interferometer arm, $r_{0}$ is the distance from the test mass to the streamline at closest approach, $r_{0} \cos \psi$ is the projection of this distance onto the ground, and $\mathbf{v}=$ constant is the velocity of the airflow.

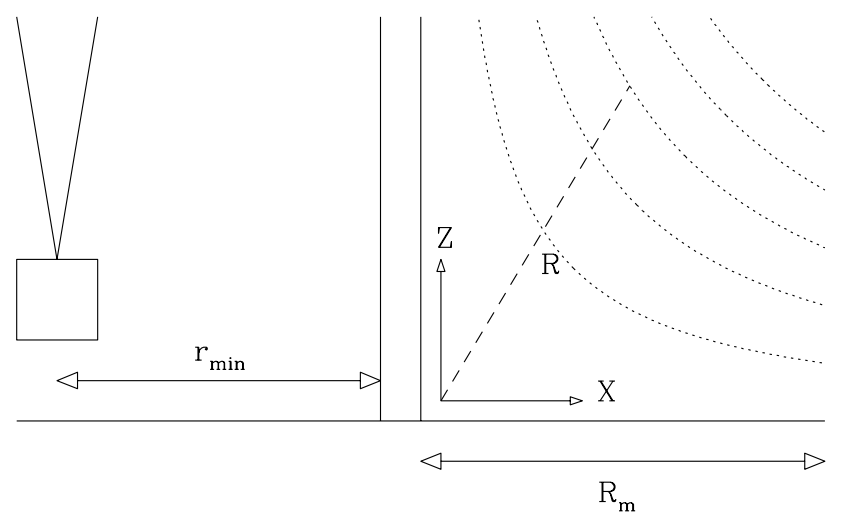

FIG. 5: Schematic of the airflow streamlines around the corner of an end station, assuming an incompressible vorticityfree flow. The dotted lines are the streamlines, $r_{\min }$ is the minimum distance that the airflow can approach the test mass, $R_{m}$ is the scale distance at which the flow velocity $v$ approaches the free-streaming speed $V$, and $X, Z$, and $R$ are coordinates used to describe the velocity field.

of order $R_{m} / V$ for all streamlines. Thus the streamlines close to the corner will contribute no more high-frequency noise than the streamlines further out, at distances of order $R_{m}$, and the spectrum should not differ greatly from the one for uniform flow, Eqs. (17), (18).

\section{Vortices}

Perhaps the most serious contenders for high-frequency atmospheric gravity gradient noise are circulating vor- tices of air near the end stations. This is somewhat stretching the assumptions of the formalism I have established, since I had previously separated the effects of the homogeneous turbulence (which establishes the temperature perturbations on various lengthscales), and wind flow (which carries these perturbations past the instrument). However, the results in this section are only expected to be good to order of magnitude anyway, in the absence of detailed hydrodynamic analysis of airflow past a particular interferometer building. I therefore apply the above formalism to a simple model for a turbulent-like flow past an interferometer end station.

For simplicity, I specialize to airflow along the axis of an interferometer arm, since these give the largest gravitational accelerations. A simple model for the turbulent flow is to take a uniform flow and then add a cycloidal motion to it. This gives $x(t) \sim v t-R \sin (v t / R)$, $z(t) \sim r_{0}-R \cos (v t / R), r(t)=\sqrt{z^{2}(t)+x^{2}(t)}$, where $R$ is the radius of the cycloidal motion, and $r_{0} \sim r_{\text {min }}$ is the distance from the test mass to the unperturbed streamline. I treat the speed $v$ along the streamline as a constant. If $R$ is also a constant, then one would expect the Fourier transform of $G(t)=x(t) / r^{3}(t)$ [Eq. (12)] to have a spike at frequency $f=v / 2 \pi R$, with a width of order $\sim v / r_{0}$. However, to give a somewhat more realistic behaviour for $R$, I treat it as growing from zero at the leading edge of the end station to some scale value $R_{0}$ over the half-length $\sim r_{\text {min }}$ of the end station: $R(t)=R_{0} \sqrt{v t / r_{\min }}$. The square-root dependence mimics the less-than-linear growth of the thickness of a boundary layer. Although quite crude, this model covers the essential features of a turbulent flow past the building: a uniform translation, accompanied by circulating 


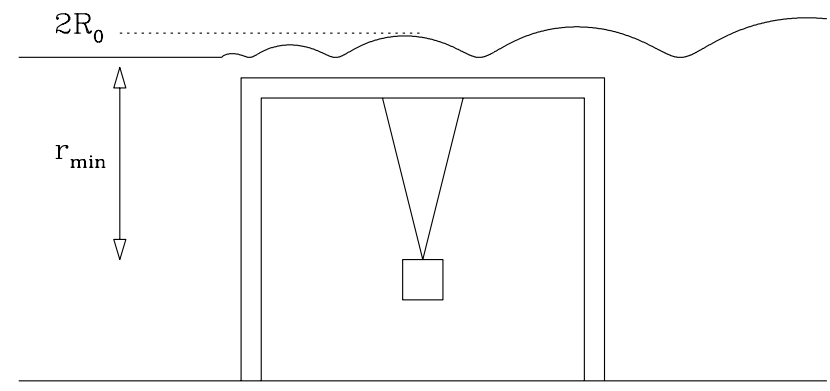

FIG. 6: Schematic of a vortex-like streamlines across the top of an end station. The quantity $r_{\min }$ is the minimum distance that the airflow can approach the test mass, and $R_{0}$ is the typical radius of circulation at this distance.

motions over a range of radii with a scale set by $R_{0}$, with both the uniform flow rate and the circulation speed set by the free-streaming airspeed $v$. A typical streamline of this type is shown in Fig. 6 .

The Fourier transform of $G(t)$ is too complicated to perform analytically, but is simple enough to compute numerically using a fast Fourier transform. The resulting $\tilde{G}(f)$ has the usual exponential cutoff with frequency scale $v / 2 \pi r_{\min }$, as for a smooth streamline, but then rises to a second peak value of $\sim(4 / v) \sqrt{R_{0}^{3} / r_{\min }^{5}}$ at a frequency $f \sim 0.06 \mathrm{v} / R_{0}$ and decreases from there as $f^{-3}$. This high-frequency tail is the power law that one would expect from the cusps on the bottom of the cycloid. Since I treat $v$ as constant, $\tilde{F}(f)=v^{p+3} \tilde{G}(f)$. The crosssectional area of streamlines that contribute significantly around this peak frequency is of order $\sim 2 r_{\min } R_{0}$. Plugging these into Eq. 10) one gets a noise spectral density of

$$
S_{h(\max )} \sim 1.5 \times 10^{6}\left(\frac{G \rho}{L T}\right)^{2} c_{T}^{2} R_{\max }^{35 / 3} r_{\min }^{-4} v^{-5}
$$

at the peak frequency of $f \sim 0.06 v / R_{0}$, where I have assumed $p=2 / 3$.

For wind speeds around $10 \mathrm{~m} / \mathrm{s}$, a cycloid radius $R_{0} \sim$ $0.06 \mathrm{~m}(v / 10 \mathrm{~m} / \mathrm{s})$ puts the noise peak at the $10 \mathrm{~Hz}$ seismic noise wall for advanced LIGO detectors. The atmospheric gravitational noise contribution from a single end station is then:

$$
\begin{aligned}
S_{h(\max )} \sim & \left(1.3 \times 10^{-49} \mathrm{~Hz}^{-1}\right)\left(\frac{c_{T}^{2}}{0.2 \mathrm{Km}^{-2 / 3}}\right) \\
& \times\left(\frac{v}{10 \mathrm{~m} / \mathrm{s}}\right)^{20 / 3}\left(\frac{r_{\min }}{5 \mathrm{~m}}\right)^{-4} .
\end{aligned}
$$

This is over five orders of magnitude below the expected advanced LIGO noise floor of $2 \times 10^{-45} \mathrm{~Hz}^{-1}$ at $10 \mathrm{~Hz}$. Gale-force winds $(v \sim 30 \mathrm{~m} / \mathrm{s})$ will bring this up to $2 \times 10^{-46} \mathrm{~Hz}^{-1}$, still an order of magnitude below the advanced LIGO noise curve. The dashed lines in Fig. 7 show the actual data from the numerical Fourier transforms for these two cases.

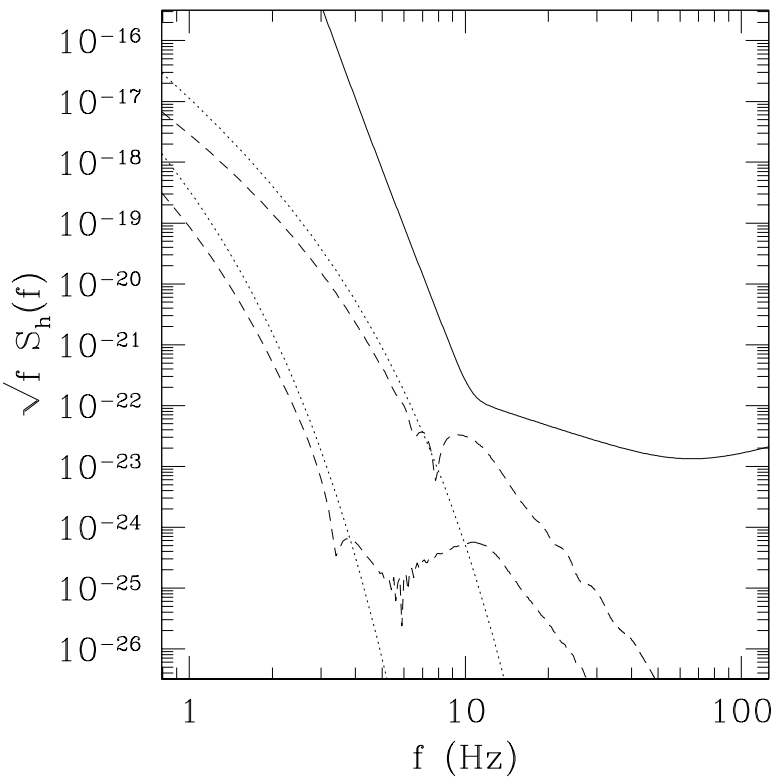

FIG. 7: Plot of dimensionless noise amplitude $\sqrt{f S_{h}(f)}$ versus frequency $f$. The solid line is the projected noise floor for advanced LIGO detectors. The two dotted lines are the gravity gradient noise levels caused by temperature perturbations advected along smooth streamlines at $10 \mathrm{~m} / \mathrm{s}$ (left curve) and $30 \mathrm{~m} / \mathrm{s}$ (right curve). The dashed lines are the noise levels caused by temperature perturbations advected along cycloidal vortices, as described in Sec. III G, at $10 \mathrm{~m} / \mathrm{s}$ (left curve) and $30 \mathrm{~m} / \mathrm{s}$ (right curve); in each case the size scales $R_{0}$ of the vortices have been tuned to maximize the noise at $10 \mathrm{~Hz}$. Even given these fine-tunings, the gravity gradient noise curve in the worst-case scenario is still nearly an order of magnitude (in amplitude) below the advanced LIGO sensitivity.

Since even the worst-case estimate is still an order of magnitude below the advanced LIGO noise floor (as well as the seismic gravity gradient noise floor in [3]), it seems unlikely that turbulent vortices will be sufficient to raise the atmospheric gravitational noise to significant levels, even given the approximations made in this analysis. However, to settle this matter definitively would require a much more sophisticated numerical analysis of the temperature perturbations and airflow past the buildings of a particular facility.

\section{SHOCKWAVES}

Although atmospheric pressure waves are unlikely to be a significant source of gravity gradient noise in interferometric gravitational-wave detectors, the sudden pressure changes caused by atmospheric shockwaves could potentially produce detectable transient signals in the detector, if such shocks occur in the vicinity of the detectors. Shocks are specifically a matter of concern because they can produce significant pressure changes over timescales less than $0.1 \mathrm{~s}$, corresponding to the lower end 
of the pass-bands of most interferometric detectors.

Consider a shock that produces a sudden jump in air pressure in the vicinity of one of the interferometer test masses: $\delta p(t)=\Delta p \Theta\left(t-t_{0}\right)$. It is a simple matter to take the Fourier transform and apply Eq. (3) to obtain:

$$
\tilde{h}(f)=\frac{G \rho c}{8 \pi^{3} \gamma L f^{4}} \frac{\Delta p}{p} i e^{2 \pi f t_{0}} \cos (\theta) C\left(2 \pi f r_{\min } / c\right) .
$$

Here, $\theta$ is the angle between the interferometer arm and the normal to the shock front. If the shock has a finite rise time $\Delta t$, we can mimic this analytically by convolving in the time domain with a Gaussian of width $\sigma \sim \Delta t$. In the frequency domain this multiplies our amplitude by a Gaussian of width $\sigma \sim 1 / \Delta t$, giving an exponential cutoff at frequencies above $1 / \Delta t$. Typical shocks from, for instance, supersonic objects have rise times on the order of a few milliseconds [8], corresponding to cutoff frequencies of a few hundred Hz. However, one expects the dominant contribution of the signal to come from much lower frequencies, before the building-size cutoff factor $C$ kicks in.

Shocks are transient phenomena that will produce signals in the detector, rather than raising the noise floor. What one would like to know is what signal-to-noise ratio the shock will produce. In general this depends on what filters one is using to search for signals, and how well these filters overlap with the signal produced by a shock. However, the signal from a shock is likely to overlap quite well with templates designed to search for generic impulsive phenomena; such templates are likely to be used in advanced interferometers as control over nonstationary instrumental noise improves. Thus it is reasonable to consider the signal-to-noise ratio $\rho$ that a matched filter would give [9]:

$$
\rho^{2}=\int_{0}^{\infty} \frac{4 \tilde{h}(f) \tilde{h}^{*}(f)}{S_{h}(f)} d f=\int_{-\infty}^{\infty} \frac{|2 f \tilde{h}(f)|^{2}}{f S_{h}(f)} d \ln f .
$$

This shows, roughly speaking, that the relative magnitude of the dimensionless signal amplitude $2|f \tilde{h}(f)|$ and the dimensionless noise amplitude $\sqrt{f S_{h}(f)}$ over a logarithmic frequency interval gives a good indication of the signal-to-noise ratio produced in the detector.

\section{A. Sonic booms}

Sonic booms caused by supersonic bodies are one example of atmospheric shocks that might affect interferometric gravitational-wave detectors. Direct shockwaves from a supersonic aircraft will typically hit the ground in a "carpet" about 15-20 km wide under the aircraft's flight path. Outside this carpet, the temperature gradient near the ground will completely reflect the shockwave before it touches down. However, shockwaves will also reflect downward off of the temperature inversion in the stratosphere and thermosphere, forming secondary and higher-order "carpets" out to many hundreds of kilometres [10]. The presence or absence of these higher-order waves can depend quite sensitively on conditions in the upper atmosphere.

A detailed prediction of these effects is beyond the scope of this paper. However, to give an indication of their potential seriousness, I will consider what would happen if a supersonic aircraft were actually to overfly the instrument at a height of several kilometres.

The characteristic profile of a sonic boom is a symmetric N-wave, consisting of a shock that increases the pressure by an amount $\Delta p$, followed by a smooth decrease in pressure of $2 \Delta p$ over a time $\Delta t$, followed by a second rising shock $\Delta p$ to restore the ambient pressure. According to Eq. (9.78) of [11], the strength of the shocks is:

$$
\frac{\Delta p}{p} \approx \frac{2^{1 / 4} \gamma}{(\gamma+1)^{1 / 2}}\left(M^{2}-1\right)^{1 / 8} \kappa \delta l^{3 / 4} r^{-3 / 4},
$$

where $\gamma$ is the adiabatic coefficient of air (1.4 at normal temperatures), $M$ is the Mach number of the aircraft (its speed divided by the sound speed), $\kappa$ is a dimensionless form factor that depends on the shape of the aircraft (typically around 1), $l$ is the length of the aircraft, $\delta$ is the ratio of the aircraft's typical thickness to its length, and $r$ is the closest distance that the aircraft came to the point of measurement. Between the two shocks, the rate of change of pressure in a direction $\mathbf{e}_{x}$ parallel to the line of flight is given, in Eq. (9.80) of [11, as:

$$
\frac{1}{p} \frac{d p}{d x} \approx \frac{\gamma}{\gamma+1} \frac{\left(M^{2}-1\right)^{1 / 2}}{M^{2}} \frac{1}{r} .
$$

The shock fronts move outward at the sound speed $c$ in the direction orthogonal to their surface, while the entire cone travels in the $\mathbf{e}_{x}$ direction along with the aircraft at a speed $M c$. The total change of pressure between the two shocks is $2 \Delta p$. From these facts and Eqs. (24) and (25), one can show that the time between the two shocks is:

$$
\Delta t \approx 2^{5 / 4}(\gamma+1)^{1 / 2} \frac{M}{\left(M^{2}-1\right)^{3 / 8}} \kappa \delta \frac{l^{3 / 4} r^{1 / 4}}{c} .
$$

On frequency scales higher than $1 / \Delta t$ (typically a few $\mathrm{Hz}$ for a supersonic aircraft a few kilometres away), the sonic boom looks like simple Heaviside shocks, giving $\delta \tilde{p}(f) \sim 1 / f$. At lower frequencies, though, the entire Nwave looks like the derivative of a $\delta$-distribution, giving $\delta \tilde{p}(f) \sim f$. Performing the Fourier transform analytically and plugging into Eq. (3), one obtains:

$$
\begin{aligned}
\tilde{h}(f)= & \frac{G \rho c}{4 \pi^{3} \gamma L} \frac{1}{f^{4}} \frac{\Delta p}{p} \cos (\theta) C\left(2 \pi f r_{\min } / c\right) \\
& \times\left[\frac{\sin (\pi f \Delta t)}{\pi f \Delta t}-\cos (\pi f \Delta t)\right] e^{2 \pi i f t_{0}},
\end{aligned}
$$

where $t_{0}$ is the time when the midpoint of the $\mathrm{N}$-wave crosses the detector. As expected, the amplitude goes 
roughly as $f^{-4}$, except for frequencies less than $1 / \Delta t$, where it goes as $f^{-1}$.

Now let us plug in some typical numbers. The numbers $G=6.67 \times 10^{-11} \mathrm{~m}^{3} \mathrm{~kg}^{-1} \mathrm{~s}^{-2}, \rho=1.3 \mathrm{~kg} \mathrm{~m}^{-3}, \gamma=1.4$, $c=332 \mathrm{~m} \mathrm{~s}^{-1}$, and $L=4000 \mathrm{~m}$ can be treated as constant. A supersonic jet aircraft might have a length of $l=10 \mathrm{~m}$, a typical diameter of $\delta l=2 \mathrm{~m}$, and be traveling at Mach $M=1.5$ at a distance of $r=10 \mathrm{~km}$ or so. Let $\cos \theta$ be 1 for an upper limit. Then $\Delta t \sim 0.2 \mathrm{~s}$, and for frequencies $f \gtrsim 10 \mathrm{~Hz}$ the dimensionless signal amplitude is:

$$
\begin{aligned}
2|f \tilde{h}(f)| \sim & 1.4 \times 10^{-19}\left(M^{2}-1\right)^{1 / 8} C\left(2 \pi f r_{\min } / c\right)\left(\frac{\delta}{0.2}\right) \\
& \times\left(\frac{l}{10 \mathrm{~m}}\right)^{3 / 4}\left(\frac{r}{10 \mathrm{~km}}\right)^{-3 / 4}\left(\frac{f}{10 \mathrm{~Hz}}\right)^{-3} \cdot(28)
\end{aligned}
$$

This is three orders of magnitude above the expected noise floor of $\sqrt{f S_{h}(f)} \sim 1.4 \times 10^{-22}$ at $10 \mathrm{~Hz}$ for advanced LIGO interferometers!

By contrast, consider a .30-calibre rifle bullet $(l \approx$ $0.025 \mathrm{~m}, \delta \approx 0.3$ ) passing at Mach 3 within $10 \mathrm{~m}$ of an interferometer test mass. (This stretches the assumption of a plane-wave shock front at the test mass, but the order of magnitude should be correct.) The bullet produces a much stronger double shock, but with a time interval $\Delta t \sim 0.5 \mathrm{~ms}$, so $1 / \Delta t \sim 2 \mathrm{kHz}$. The low-frequency tail of this signal will have dimensionless amplitude:

$$
\begin{aligned}
2|f \tilde{h}(f)| \sim & 1.8 \times 10^{-23} \frac{M^{2}\left(M^{2}-1\right)^{-5 / 8}}{2.5} C\left(2 \pi f r_{\min } / c\right) \\
\times & \left(\frac{\delta}{0.3}\right)^{3}\left(\frac{l}{0.025 \mathrm{~m}}\right)^{9 / 4} \\
& \times\left(\frac{r}{10 \mathrm{~m}}\right)^{-1 / 4}\left(\frac{f}{10 \mathrm{~Hz}}\right)^{-1} .
\end{aligned}
$$

This is nearly an order of magnitude below the dimensionless noise amplitude in advanced LIGO, and therefore too small to be of any serious concern.

Fig. 8 shows more complete gravity gradient signal spectra computed using Eq. (27) with the above parameters for a supersonic aircraft and rifle bullet. These are plotted along with the anticipated dimensionless noise amplitude for advanced LIGO detectors.

\section{B. Vetoing shockwave signals}

While atmospheric shockwaves are a potential source of spurious signals in gravitational-wave detectors, they are easy to veto using environmental sensors. One need simply place infrasound microphones outside the buildings and test-mass vacuum enclosures. If these sensors detect a pressure change of more than a millibar over timescales of 50-100 milliseconds, then one might expect spurious signals with dimensionless amplitude of $\sim 10^{-22}$ in the $10-20 \mathrm{~Hz}$ frequency range. The stretch of data containing the potential spurion can then be discarded.

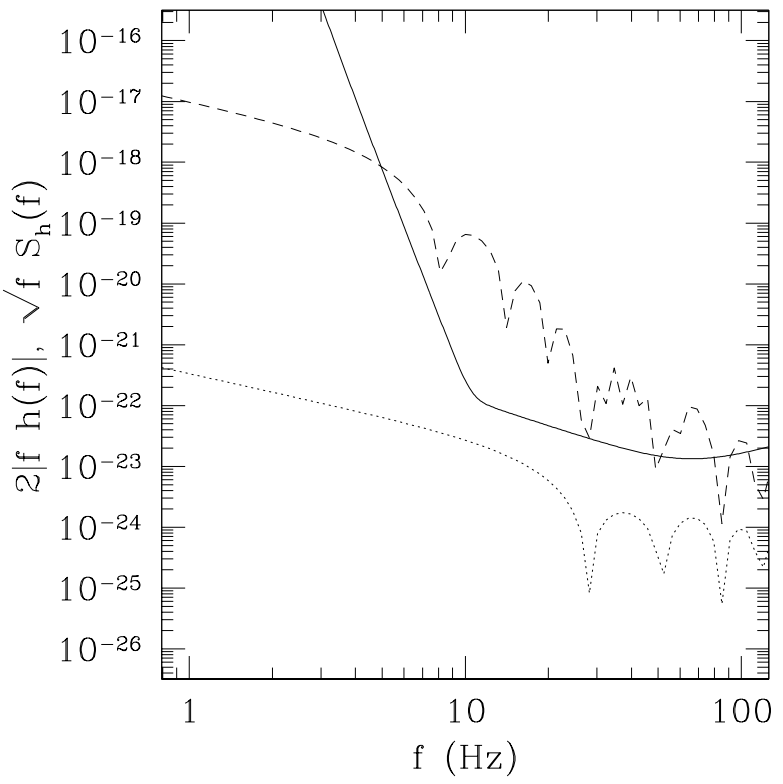

FIG. 8: Plot comparing dimensionless signal strengths $2|f \tilde{h}(f)|$ of gravity gradients from sonic booms with the dimensionless interferometer noise amplitude $\sqrt{f S_{h}(f)}$, as a function of frequency. The solid line is the projected noise amplitude for advanced LIGO detectors. The dotted line is the signal from a .30 calibre bullet traveling at Mach 3 within $10 \mathrm{~m}$ of the end station; the dashed line is the signal from a $10 \mathrm{~m}$ long aircraft traveling at Mach 1.5 at a distance of $10 \mathrm{~km}$. The aircraft's sonic boom can produce a detectable gravity gradient signal.

Alternatively, if the same shock profile is detected in an array of at least three sound sensors, then one can determine the direction of propagation of the shock and predict the actual induced test-mass motions. The spurious signal could then be subtracted out of the data stream. This is a much trickier procedure, and would only be necessary in the unlikely event that significant amounts of data were being corrupted.

In either case, it is clear that infrasound sensors will be important environmental monitors for advanced interferometric detectors.

\section{HIGH-SPEED OBJECTS}

Another potential source of spurious signals in the interferometer is the gravity gradient caused by the motion of an individual massive object past the interferometer end station, or the collision of such an object with the end station. The latter is particularly serious, since the sudden deceleration of the object can produce a signal at high frequencies. The issue of human-generated gravity gradient noise has been addressed in [9], but there are other sources outside the facility that must be considered, such as stray bullets and wind-borne debris. In 
particular, the Hanford LIGO facility is plagued by tumbleweeds, which can produce non-negligible gravity gradient signals.

The general formula for the spurious gravitationalwave signal produced by a moving object is:

$$
\tilde{h}(f)=\frac{G M}{4 \pi^{2} L f^{2}} \int_{-\infty}^{\infty} \frac{x(t)}{r^{3}(t)} e^{2 \pi i f t} d t,
$$

where $M$ is the mass of the object, $r(t)$ is its distance from the test mass as a function of time, and $x(t)$ is its distance from the end mass in the direction parallel to the interferometer arm. For an object traveling parallel to the ground in a straight line at speed $v$, Eq. (30) becomes [as in Eq. (14)]:

$$
\begin{aligned}
\tilde{h}(f)=\frac{G M}{L v^{2} \pi f} & {\left[K_{0}\left(\frac{2 \pi f r_{\min }}{v}\right) \cos \theta\right.} \\
& \left.\quad-i K_{1}\left(\frac{2 \pi f r_{\min }}{v}\right) \sin \theta \cos \psi\right] e^{2 \pi i f t_{0}}
\end{aligned}
$$

where $\theta$ is the angle between the line of motion and the interferometer arm, $r_{\min }$ is the distance of closest approach between the object and the test mass, $\psi$ is the angle projecting this distance onto the ground, and $t_{0}$ is the time of closest approach. $K_{0}$ and $K_{1}$ are modified Bessel functions of the second kind of order 0 and 1 , respectively.

Under moderately windy conditions (wind speeds up to $15 \mathrm{~m} / \mathrm{s}$ or so), tumbleweeds at the Hanford LIGO facility will bounce along the ground at $5-10 \mathrm{~m} / \mathrm{s}$. In stronger winds, the tumbleweeds become airborne, with speeds approaching the wind speed; they can fly right over the LIGO buildings, or impact with considerable force. The same may be true of wind-borne debris at other interferometer facilities. However, the value of $r_{\text {min }}$ is usually at least $5 \mathrm{~m}$, so for frequencies above $10 \mathrm{~Hz}$ one has $2 \pi f r_{\min } / v \gtrsim 10$ even for very strong winds $(v \sim 30 \mathrm{~m} / \mathrm{s})$. In this regime the Bessel functions are exponentially damped, so these objects will not produce significant gravity gradient signals simply by blowing past the instrument. A rifle bullet, on the other hand, might be moving around $1000 \mathrm{~m} / \mathrm{s}$, putting us in the small-argument regime of the Bessel functions, where $K_{0}(x) \sim-\ln (x)$ and $K_{1}(x) \sim x^{-1}$. Taking the most dangerous geometry $\theta=\pi / 2, \psi=0$, and assuming a bullet mass of around 5 grammes, this gives a dimensionless signal amplitude near $10 \mathrm{~Hz}$ of:

$$
\begin{aligned}
2|f \tilde{h}(f)| \sim & 1.6 \times 10^{-22}\left(\frac{M}{5 \mathrm{~g}}\right)\left(\frac{1000 \mathrm{~m} / \mathrm{s}}{v}\right) \\
& \times\left(\frac{5 \mathrm{~m}}{r_{\text {min }}}\right)\left(\frac{10 \mathrm{~Hz}}{f}\right) .
\end{aligned}
$$

This gives a signal-to-noise ratio of about 1 at $10 \mathrm{~Hz}$, a bit below the detectable threshold. In fact, even if one fine-tunes the bullet speed $v$, the largest signal-to-noise ratio that one can get at $10 \mathrm{~Hz}$ is about 2 , for a speed of around $250 \mathrm{~m} / \mathrm{s}$. Since events with signals less than about 5 times the noise will probably be ignored in any case, one can conclude that objects flying past a test mass are not likely to be serious sources spurious events.

If an object does not pass smoothly by the interferometer but instead collides with an end station, the signal at $\sim 10 \mathrm{~Hz}$ can be large even for slow-moving objects: it is the deceleration time, not the end-station-crossing time, that sets the frequency scale of the signal. Suppose an object collides end-on with the end station at a speed $v$, coming to a stop within a distance $d$ at constant acceleration. Let $t=0$ denote the time that the object comes to rest. The motion of the object is then given by:

$$
r(t)=x(t)=\left\{\begin{array}{lc}
r_{\min }-d-v t & t \leq-2 d / v \\
r_{\min }+(v t)^{2} / 4 d & -2 d / v \leq t \leq 0 \\
r_{\min } & t \geq 0
\end{array} .\right.
$$

The Fourier transform of $x(t) / r^{3}(t)=1 / r^{2}(t)$ is tricky to do analytically, so I have relied on numerical fast Fourier transforms, and then made approximate analytic fits to the result. However, one can qualitatively predict the shape of the signal in frequency space. The function $1 / r^{2}(t)$ starts out near zero and then slowly rises over a timescale $r_{\min } / v$ to a value $r_{\min }^{2}$, then quickly levels off at that value over a timescale $d / v$. So on frequency scales $\ll v / r_{\min }, 1 / r^{2}(t)$ looks like a step function, whose Fourier transform goes as $1 / f$. On frequency scales $\geq v / r_{\min }$ but $\ll v / d$, one sees the deceleration as a cusp (discontinuous first derivative), giving a Fourier transform that goes as $1 / f^{2}$. On frequency scales $\geq v / d$, the deceleration appears smooth, but the onset of deceleration in sudden, giving a $1 / f^{3}$ behaviour. The following gives a good fit to the numerical Fourier transform:

$$
\begin{aligned}
\left|\int_{-\infty}^{\infty} \frac{e^{2 \pi i f t}}{r^{2}(t)} d t\right| \sim \frac{1}{v r_{\min }} & {\left[5.9\left(\frac{f r_{\min }}{v}\right)+14\left(\frac{f r_{\min }}{v}\right)^{2}\right.} \\
& \left.+59\left(\frac{f^{3} r_{\min }^{2} d}{v^{3}}\right)\right]^{-1} \cdot
\end{aligned}
$$

The two breakpoints separating the three branches are at frequencies $f_{1}=0.4 v / r_{\min }$ and $f_{2}=0.24 v / d$. More precisely, since the deceleration occurs over a well-defined time $2 d / v$, the third branch of the Fourier transform is oscillatory with nodes every $v / 2 d$ in frequency space; the functional fit in Eq. (34) is an envelope containing these oscillations.

For a $5 \mathrm{~g}$ bullet striking an end station at $1000 \mathrm{~m} / \mathrm{s}$, the signal at $10 \mathrm{~Hz}$ is dominated by the low-frequency tail regardless of stopping distance. The dimensionless signal amplitude is then:

$$
2|f \tilde{h}(f)| \sim 3 \times 10^{-22}\left(\frac{M}{5 \mathrm{~g}}\right)\left(\frac{10 \mathrm{~Hz}}{f}\right)^{2}\left(\frac{5 \mathrm{~m}}{r_{\min }}\right)^{2} .
$$

The signal amplitude is about the same as for a passing bullet, although the dependence on $f$ (and $r_{\min }$ and $v$ ) is different. For a tumbleweed or other wind-borne 
object, by contrast, the sudden deceleration can create significant high-frequency noise. A typical tumbleweed at Hanford has a mass of $M=0.1 \mathrm{~kg}$ and a diameter of $0.4 \mathrm{~m}$, and can be compressed by about half that amount $(d=0.2 \mathrm{~m})$. Larger weeds can be twice as large in diameter, putting their masses in the $0.5-1 \mathrm{~kg}$ range 12 . For moderate to high wind speeds $(v=10-30 \mathrm{~m} / \mathrm{s})$, the signal at frequencies above $10 \mathrm{~Hz}$ is dominated by the second branch of Eq. (34), giving a dimensionless signal amplitude of:

$$
\begin{aligned}
2|f \tilde{h}(f)| \sim 5 \times & 10^{-21}\left(\frac{M}{1 \mathrm{~kg}}\right)\left(\frac{v}{10 \mathrm{~m} / \mathrm{s}}\right) \\
& \times\left(\frac{10 \mathrm{~Hz}}{f}\right)^{3}\left(\frac{5 \mathrm{~m}}{r_{\min }}\right)^{3} .
\end{aligned}
$$

Thus even a "typical" $0.1 \mathrm{~kg}$ weed at these speeds will produce a signal-to-noise ratio of around 4 at $10 \mathrm{~Hz}$ in advanced LIGO interferometers, which is in danger of being interpreted as a real gravitational-wave event. In a more extreme case, a $1 \mathrm{~kg}$ tumbleweed blown airborne by a strong $30 \mathrm{~m} / \mathrm{s}$ wind will produce a signal 100 times higher than the noise at $10 \mathrm{~Hz}$, which is easily detectable.

Fig. 9 shows the signal spectra for the objects discussed above, plotted against the dimensionless noise amplitude expected in advanced LIGO detectors. Since tumbleweeds are a potential source of spurious detectable events, one should consider ways to reduce the tumbleweed gravity gradient noise. Fortunately, the signal goes as $r_{\text {min }}^{-3}$, so a simple fence preventing the weeds from approaching the end station should be sufficient. A fence $30 \mathrm{~m}$ out from the end station will reduce the signal-tonoise ratio to 1 for tumbleweed masses up to $1 \mathrm{~kg}$ and speeds up to $30 \mathrm{~m} / \mathrm{s}$, reducing the risk of spurious events.

\section{CONCLUSIONS}

This paper has studied two sources of background gravity gradient noise, from infrasonic atmospheric pressure waves and from wind-advected temperature perturbations, in order to determine whether they constitute a limiting noise floor for interferometric gravitational-wave detectors - in particular, for the "advanced" LIGO detectors projected in [1]. The paper also analyzed two sources of gravity gradient signals, from transient atmospheric shockwaves and from massive airborne bodies, to determine whether they would constitute detectable spurious events in these interferometers. The following summarizes the results and suggests possible further work that may need to be done.

Current estimates suggest that infrasonic pressure waves will not be a significant source of gravity gradient noise, being over two orders of magnitude below the advanced LIGO noise floor at $10 \mathrm{~Hz}$. Nonetheless, these estimates are not based on actual noise measurements at an interferometer site, so infrasound measurements at these sites are recommended to confirm them. Further

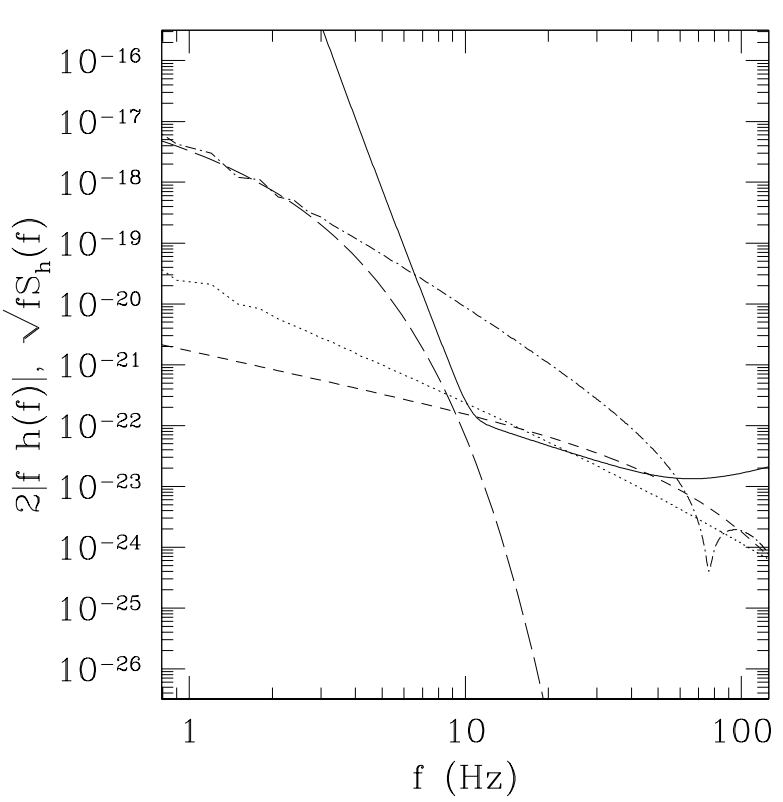

FIG. 9: Plot comparing dimensionless signal strengths $2|f \tilde{h}(f)|$ of gravity gradients from airborne objects with the dimensionless interferometer noise amplitude $\sqrt{f S_{h}(f)}$, as a function of frequency. The solid line is the projected noise amplitude for advanced LIGO detectors. The short-dashed line is the signal from a .30 calibre bullet passing next to the interferometer end station at $1000 \mathrm{~m} / \mathrm{s}$, while the dotted line is the signal from that same bullet colliding with the end station. The long-dashed line is the signal from a large $1 \mathrm{~kg}$ tumbleweed passing next to the end station at $30 \mathrm{~m} / \mathrm{s}$, while the dot-dashed line is the signal from that same tumbleweed colliding with the end station, assuming that it compresses by $20 \mathrm{~cm}$ on impact. The tumbleweed mass and speed are both near the upper end of the expected range, representing the greatest danger of spurious signals in the detector; evidently these tumbleweed signals can be detected quite easily by advanced LIGO detectors.

empirical studies might also analyze the specific effects of building shapes and of infrasound coherence lengths on the noise spectrum, although these refinements would likely only serve to reduce noise estimates above $15 \mathrm{~Hz}$ or so.

Wind-advected temperature perturbations, although the dominant source of atmospheric density fluctuations, do not produce significant high-frequency gravity gradient noise, due to the long times that any particular pocket of warm or cool air spends in the vicinity of an interferometer test mass. A possible exception is when the airflow forms vortices around the interferometer buildings, since this will produce a noise spectrum peaked around the typical vortex circulation frequencies near the test mass. The current crude analysis of these effects suggests that the noise is still an order of magnitude below the advanced LIGO noise floor at $10 \mathrm{~Hz}$ even in the worst-case scenarios, but the model could be improved significantly. Numerical models of the airflow and of temperature per- 
turbations near an interferometer building may be required to settle this issue definitively.

Gravity gradients from atmospheric shockwaves are potentially serious sources of spurious signals in interferometric gravitational-wave detectors. For instance, the sonic boom from a supersonic aircraft overflying an advanced LIGO detector could produce signal-to-noise ratios of several hundred. Although such overflights are expected to be rare or nonexistent, they point out the potential seriousness of shocks from weaker or more distant sources, even if the signals are several orders of magnitude smaller. It is therefore strongly recommended that advanced interferometric detectors include infrasonic detectors as environmental monitors. Such sensors could easily be employed to veto spurious atmospheric gravitygradient events.

Gravity gradients from wind-borne objects such as tumbleweeds are another possible source of spurious events in gravitational-wave detectors, if these objects are allowed to collide with the buildings housing the interferometers. Fences or other structures should be used to keep these objects at least 30 metres from the test masses, in order to eliminate the risk of spurious signals.

Obviously the number of things that can affect interferometric detectors through gravity gradient forces is immense; I have considered here only the few sources that I considered the most worrisome. I encourage other researchers to consider the implications of this oftenneglected effect.

\section{Acknowledgments}

This work was supported by NSF grant PHY-9424337. I would like to thank Kip Thorne for his help and support throughout this project, as well as Brad Sturtevant, Brian Kern, and Scott Hughes for their insightful discussions about atmospheric phenomena. I am especially grateful to Fred Raab for collecting the vital statistics of Hanford tumbleweeds.

\section{APPENDIX A: THE TEMPERATURE NOISE SPECTRUM}

This appendix presents a more rigorous mathematical derivation of Eq. (10) used in Sec. III.

Consider a time-varying field of temperature perturbations $\delta T(\mathbf{r}, t)$ about some average temperature $T$. This produces a gravitational perturbation $g_{x}(t)=$ $\int d V \operatorname{Goxr}^{-3}(\delta T / T)$, where $\rho$ is the average air density, and the $x$-axis is along the interferometer arm. The spectral density of gravity gradient noise $S_{g}(|f|)$ is given by twice the Fourier transform of the gravity autocorrelation $C_{g}(\tau)=\langle g(t) g(t+\tau)\rangle$. Thus:

$$
S_{g}(|f|)=2\left(\frac{G \rho}{T}\right)^{2} \int_{-\infty}^{\infty} d \tau \int d V \int d V^{\prime} \frac{x x^{\prime}}{r^{3}\left(r^{\prime}\right)^{3}}
$$

$$
\left\langle\delta T(\mathbf{r}, t) \delta T\left(\mathbf{r}^{\prime}, t+\tau\right)\right\rangle e^{2 \pi i f \tau} .
$$

The temperature noise measured at a point $\mathbf{r}_{0}$, on the other hand, is given simply by:

$$
S_{T}(|f|)=\int_{-\infty}^{\infty} d \tau\left\langle\delta T\left(\mathbf{r}_{0}, t\right) \delta T\left(\mathbf{r}_{0}, t+\tau\right)\right\rangle e^{2 \pi i f \tau}
$$

On sufficiently small scales, the temperature perturbations in the Earth's turbulent boundary layer can be treated as homogeneous and isotropic. The expected squared temperature difference between two points is then a function only of their separation: $\langle[\delta T(\mathbf{r})-\delta T(\mathbf{r}+$ $\left.\Delta \mathbf{r})]^{2}\right\rangle=D_{T}(\|\Delta \mathbf{r}\|)$. The function $D_{T}(\Delta r)$ is called the temperature structure function of the atmosphere, and for small $\Delta r$ reduces to a power law $D_{T}(r)=c_{T}^{2} \Delta r^{p}$. If a wind with speed $v$ blows these perturbations past a measuring station, the temperature autocorrelation is $\left\langle\delta T\left(\mathbf{r}_{0}, t\right) \delta T\left(\mathbf{r}_{0}, t+\tau\right)\right\rangle=\sigma_{T}^{2}-(1 / 2) c_{T}^{2}(v \tau)^{p}$ for small $\tau$, where $\sigma_{T}^{2}$ is the mean squared temperature fluctuation. This results in a high-frequency power law tail:

$$
S_{T}(|f|)=c_{T}^{2} v^{p}(2 \pi f)^{-(p+1)} \Gamma(p+1) \sin (p \pi / 2) .
$$

Turbulent mixing theory, as well as micrometeorological measurements of $S_{T}(|f|)$, show that the value of $p$ is normally $2 / 3$, characteristic of a type of turbulence known as Kolmogorov turbulence. See, for example, [5] for discussion of this type of turbulent mixing, also [6] and references therein.

We are interested in $S_{g}(|f|)$, which is somewhat trickier to calculate than $S_{T}(|f|)$, since it involves a correlation between points separated in space as well as time. However, chaotic turbulence will almost certainly destroy high-frequency correlations between widely separated points, so the high-frequency behaviour of $S_{g}(|f|)$ will come from correlations between nearby points. That is, the high-frequency support of $\left\langle\delta T(\mathbf{r}, t) \delta T\left(\mathbf{r}^{\prime}, t+\tau\right)\right\rangle$ will come from those points $\mathbf{r}^{\prime}$ at time $t+\tau$ whose fluid elements were near $\mathbf{r}$ at time $t$.

Consider two fluid elements moving along paths $S$ and $S^{\prime}$ passing through $\mathbf{r}$ and $\mathbf{r}^{\prime}$, respectively. This is shown schematically in Fig. 10. Let $r_{0}$ be the distance from $\mathbf{r}$ to the nearest point on $S^{\prime}$, and let $\tau_{0}$ be the time it would take a pocket of air at $\mathbf{r}^{\prime}$ to be carried to this point on $S^{\prime}$. In order for these points $\mathbf{r}$ and $\mathbf{r}^{\prime}$ to contribute to the high-frequency component of the spectrum, the distance $r_{0}$ must be fairly small, of the order $v / f$ where $v$ is the wind speed past $\mathbf{r}$. I treat the streamlines as relatively straight over these scales, in which case the temperature perturbations at $(\mathbf{r}, t)$ and $\left(\mathbf{r}^{\prime}, t+\tau\right)$ correspond to physical pockets of air separated by a distance $\sqrt{r_{0}^{2}+v(\mathbf{r})^{2}\left(\tau-\tau_{0}\right)^{2}}$, for $\tau$ near $\tau_{0}$. Assuming that the $\tau$-dependence of the correlation function is due entirely to this advection, the correlation function can be written explicitly as:

$$
\left\langle\delta T(\mathbf{r}, t) \delta T\left(\mathbf{r}^{\prime}, t+\tau\right)\right\rangle=\sigma_{T}^{2}-\frac{c_{T}^{2}}{2}\left[r_{0}^{2}+v^{2}\left(\tau-\tau_{0}\right)^{2}\right]^{p / 2},
$$


where typically $p \approx 2 / 3$.

This term contains the entire $\tau$-dependence of the gravity perturbation in Eq. (A1), so the first integral I do is the Fourier integral over $\tau$. This integral has the form $\int_{-\infty}^{\infty}\left(\beta^{2}+x^{2}\right)^{\nu-1 / 2} e^{i a x} d x=2 \pi^{-1 / 2}(2 \beta / a)^{\nu} \cos (\pi \nu) \Gamma(\nu+$ $1 / 2) K_{-\nu}(a \beta)$. Formally the integral diverges for $\nu \geq 1 / 2$, but the closed-form expression remains approximately correct for large $a$ provided the integrand is cut off smoothly for large $x \gg 1 / a$. Physically this corresponds to the fact that a smooth, large-scale cutoff of the temperature correlations in Eq. (A4) will not affect the high-frequency component of the temperature noise. For horizontal winds near the ground the spatial correlation function is cut off on horizontal distance scales of $\sim 50$ times the fluid elements' altitude $z$ above ground, giving a low-frequency cutoff around $\sim 0.02 v / z$ (Fig. 1.A4 of [5]). Typically this will be below the relevant frequency range for interferometric detectors; I ignore it to obtain pessimistic (upper-limit) noise estimates. The high-frequency noise tail is then:

$$
\begin{aligned}
S_{g}(|f|)= & \left(\frac{G \rho}{T}\right)^{2} \int d V \int d V^{\prime}\left[\frac{x x^{\prime}}{r^{3}\left(r^{\prime}\right)^{3}}\right. \\
\times & c_{T}^{2} v^{p}(\sqrt{2} \pi f)^{-(p+1)} a(p)\left(\frac{2 \pi f r_{0}}{v}\right)^{(p+1) / 2} \\
& \left.\times K_{(p+1) / 2}\left(2 \pi f r_{0} / v\right) e^{2 \pi i f \tau_{0}}\right]
\end{aligned}
$$

where $a(p)=-2 \pi^{-1 / 2} \cos (\pi[p+1] / 2) \Gamma([p+2] / 2) \approx 0.873$ for $p=2 / 3$.

Next is the integral over $d V^{\prime}$. The exponential decay of the Bessel function $K_{(p+1) / 2}$ restricts the support of this integral to values $r_{0} \lesssim v / 2 \pi f$, representing the fact that high-frequency fluctuations can only arise from the rapid change of the spatial correlation function over small lengthscales. This range in $r_{0}$ defines a narrow bundle of streamlines $S^{\prime}$ about the streamline $S$ passing through r. I assume that the size of this bundle is less than the distance from the test mass to the bundle, so that the values of $x^{\prime}$ and $r^{\prime}$ on a given $S^{\prime}$ can be replaced with the nearby values on $S$. Now for points near $\mathbf{r}$ the volume element $d V^{\prime}$ can be written in terms of the new parameters $r_{0}$ and $\tau_{0}$ as $d V^{\prime}=2 \pi r_{0} d r_{0} v(\mathbf{r}) d \tau_{0}$. Since the airflow, being very subsonic, is nearly incompressible, the volume element retains this form for all points along the bundle: if $v\left(\mathbf{r}^{\prime}\right)$ decreases below $v(\mathbf{r})$, for instance, the length element $v\left(\mathbf{r}^{\prime}\right) d \tau_{0}$ will decrease, but the cross-section of the bundle (i.e., the relevant range of $2 \pi r_{0} d r_{0}$ ) will increase to compensate. Plugging in this volume element and integrating over $r_{0}$, one obtains:

$$
\begin{aligned}
S_{g}(|f|) & =\left(\frac{G \rho}{T}\right)^{2} \int d V \int v d \tau_{0}\left[\frac{x x^{\prime}}{r^{3}\left(r^{\prime}\right)^{3}} c_{T}^{2} v^{p}(\pi f)^{-(p+1)}\right. \\
& \left.\times a(p) 2 \pi \Gamma([p+3] / 2)\left(\frac{v}{2 \pi f}\right)^{2} e^{2 \pi i f \tau_{0}}\right] . \quad(\mathrm{A} 6)
\end{aligned}
$$

Let $t^{\prime}$ be a new time coordinate denoting the time it takes for an air pocket to reach $\mathbf{r}^{\prime}$ from some fixed reference plane that crosses all streamlines orthogonally, and $t$ be the corresponding coordinate for the point $\mathbf{r}$. Then $\tau_{0}$ is just $t^{\prime}-t$, and the volume element $d V$ can be written as $w d t d A$, where $d A$ is a cross-sectional area element on the reference plane, and $w$ is the wind speed across that area element. Plugging this in, and ignoring the spatial separation between $S$ and $S^{\prime}$, one obtains:

$$
\begin{aligned}
S_{g}(|f|)= & \left(\frac{G \rho}{T}\right)^{2} c_{T}^{2}(\pi f)^{-(p+3)} a(p)(\pi / 2) \Gamma([p+3] / 2) \\
\times & \int_{\{S\}} w d A\left(\int_{-\infty}^{\infty} \frac{x}{r^{3}} v^{p+3} e^{-2 \pi i f t} d t\right) \\
& \left(\int_{-\infty}^{\infty} \frac{x^{\prime}}{\left(r^{\prime}\right)^{3}} e^{2 \pi i f t} d t^{\prime}\right),
\end{aligned}
$$

where I have used $\int_{\{S\}}$ to denote an integral over the entire reference plane; i.e., over all streamlines $S$. 


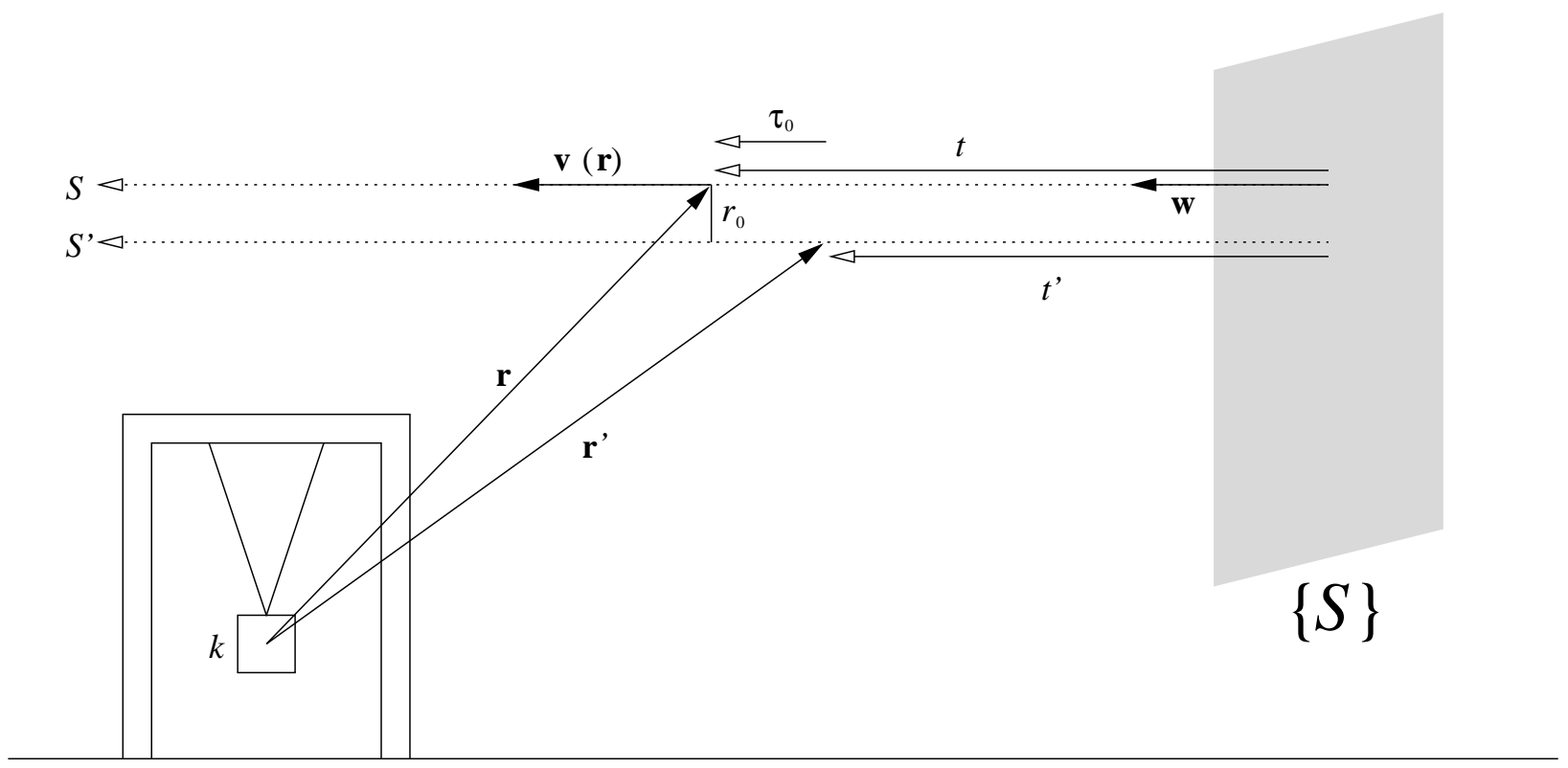

FIG. 10: Schematic showing the parameters used to describe streamlines of air flowing past an interferometer test mass. $\{S\}$ denotes a plane intersecting all streamlines; $S$ and $S^{\prime}$ are two such streamlines passing through the points $\mathbf{r}$ and $\mathbf{r}^{\prime}$, respectively, where the coordinate origin is centered on the test mass $k$. The distance between the streamlines, measured at $\mathbf{r}$, is $r_{0}$. The positions of the points $\mathbf{r}$ and $\mathbf{r}^{\prime}$ along the streamlines $S$ and $S^{\prime}$ are parameterized by the times $t$ and $t^{\prime}$ that it would take for a pocket of air to move from $\{S\}$ to $\mathbf{r}$ or $\mathbf{r}^{\prime} ; \tau_{0}$ is the difference $t-t^{\prime} . \mathbf{v}(\mathbf{r})$ is the wind velocity along $S$ through the point $\mathbf{r}$; w is the wind velocity through the plane $\{S\}$.

The noise in the gravitational-wave signal $h(t)$ due to the gradients at a given test mass is $S_{h}(|f|)=$ $(2 \pi f)^{-4} S_{g}(|f|) / L^{2}$, where $L$ is the interferometer arm length. The noise at each test mass adds incoherently to the total signal. Combining these with Eq. (A7) yields the result given in Eq. (10).
[1] A. Abramovici, W. E. Althouse, R. W. P. Drever, Y. Gürsel, S. Kawamura, F. J. Raab, D. Shoemaker, L. Siewers, R. E. Spero, K. S. Thorne, R. E. Vogt, R. Weiss, et al., Science 256(5055), 325 (1992).

[2] P. R. Saulson, Phys. Rev. D. 30(4), 732 (1984).

[3] S. A. Hughes and K. S. Thorne, Phys. Rev. D. 58(12), 122002 (1998), gr-qc/9806018.

[4] E. S. Posmentier, J. Geophys. Res. 79(12), 1755 (1974).

[5] N. E. Busch, in Workshop on Micrometeorology, edited by D. A. Haugen (American Meteorological Society, Boston, 1972), chap. 1, pp. 1-65.

[6] C. E. Coulman, Ann. Rev. Astron. Astrophys. 23, 19 (1985).
[7] L. D. Landau and E. M. Lifshitz, Fluid Mechanics (Pergamon Press, New York, 1959).

[8] W. D. Hayes, Ann. Rev. Fluid Mech. 3, 269 (1971).

[9] K. S. Thorne and C. J. Winstein, Phys. Rev. D. 60(12), 082001 (1999), gr-qc/9810016.

[10] N. K. Balachandran, W. L. Donn, and D. H. Rind, Science 197, 47 (1977).

[11] G. B. Witham, Linear and Nonlinear Waves (Wiley, New York, 1974).

[12] F. Raab, private communication (1999).

[13] Specifically, I will be using the noise curve in Fig. 10 of [1], not Fig. 7, whose suspension thermal noise is a factor of 3 too small. 